\title{
Deaf blind hypopigmentation syndrome, Yemenite type
}

INSERM

\section{Source}

INSERM. (1999). Orphanet: an online rare disease and orphan drug data base. Deaf blind hypopigmentation syndrome, Yemenite type. ORPHA:3214

Yemenite deaf-blind hypopigmentation syndrome is an exceedingly rare genetic disorder characterized by cutaneous pigmentation anomalies, ocular disorders and hearing loss. 